\title{
Significance of Uracil/Tegafur for Preventing Intravesical Recurrence of Non-Muscle Invasive Urothelial Carcinoma of the Bladder
}

\author{
Ken-ichi Harada Hideaki Miyake Tomoaki Terakawa Masato Fujisawa \\ Division of Urology, Kobe University Graduate School of Medicine, Kobe, Japan
}

\section{Key Words}

Non-muscle invasive urothelial carcinoma of the bladder . Uracil/tegafur $\cdot$ Recurrence $\cdot$ Angiogenesis $\cdot \nu$-butyrolactone

\begin{abstract}
Background:The objective of this study was to assess the role of uracil/tegafur (UFT) and its metabolite $\gamma$-butyrolactone $(G B L)$, a potent inhibitor of angiogenesis, in the prevention of intravesical recurrence in patients with non-muscle invasive urothelial carcinoma of the bladder (NMIUCB). Patients and Methods: This study included 48 patients with NMIUCB following complete transurethral resection who were randomly divided into 27 receiving UFT therapy (group A) and 21 without any adjuvant therapies (group B). Serum levels of $\mathrm{GBL}$, vascular endothelial growth factor, basic fibroblast growth factor, platelet-derived growth factor and interleukin-8 were measured. Results: There was no significant difference in the intravesical recurrence-free survival between groups A and B. Despite the lack of significant differences in serum levels of vascular endothelial growth factor, basic fibroblast growth factor, platelet-derived growth factor and interleukin-8, serum GBL in group A was significantly greater than in group B. Multivariate analysis identified tumor size as an independent predictor of intravesical recurrence irrespective of the other factors examined. Conclusions: Despite the significant induction of GBL, adjuvant UFT therapy failed to show a preventive effect on intravesical recurrence of NMIUCB. Therefore, we should consider enhancing the anti-angiogenic effect of GBL using an alternative administration schedule of UFT.

Copyright $\odot 2012$ S. Karger AG, Basel
\end{abstract}

\section{KARGER}

Fax +4161306 1234

E-Mail karger@karger.ch

www.karger.com
(C) 2012 S. Karger AG, Basel

1015-9770/12/0061-0027\$26.00/0

Accessible online at:

www.karger.com/cur

\section{Introduction}

Approximately $80 \%$ of patients with newly developed urothelial carcinoma of the bladder are pathologically diagnosed as having non-muscle invasive tumors that are limited to the urothelium or infiltrate no deeper than the lamina propria. Complete transurethral resection (TUR) of the visible tumor burden is currently regarded as the standard approach for patients with non-muscle invasive urothelial carcinoma of the bladder (NMIUCB), and the prognosis of such patients is generally favorable, achieving 5-year survival rate greater than $80 \%$ [1]. Several previous studies, however, reported that intravesical recurrence following TUR occurred in 30 to $80 \%$ of patients with NMIUCB [1-3]. Therefore, intensive efforts have been made to develop adjuvant therapy that can effectively prevent postoperative intravesical recurrence of NMIUCB.

To date, intravesical therapy has been widely performed to delay or prevent intravesical recurrence of NMIUCB following TUR. Commonly used agents for intravesical instillation include immunotherapy with bacille Calmette-Guérin and chemotherapy with mitomycin $\mathrm{C}$, adriamycin, epirubicin and gemcitabine $[4,5]$. Shelly et al. [5] recently carried out a systemic review of randomized trials associated with intravesical therapy against NMIUCB, and concluded that an immediate postTUR instillation of a chemotherapeutic agent is effective in reducing intravesical recurrence, and further intravesical induction as well as maintenance therapy with bacille Calmette-Guérin are recommended for high-risk 
patients. However, there has been no reliable evidence that any agent for intravesical therapy is able to improve overall survival. Furthermore, several investigators suggested that the effectiveness of the routine instillation of an intravesical agent is likely to remain unresolved pending more critical evaluations, and considering important issues associated with this therapy, such as complications and cost $[6,7]$.

The combination of uracil and tegafur at a molecular ratio of 4:1, also referred to as UFT, is an orally available anticancer agent. Uracil inhibits the degradation of fluorouracil to inactivate the metabolite through biochemical modulation, and therefore, UFT exerts its activity more specifically within tumor tissues than that in normal tissues [8]. To date, there have been a number of studies showing the efficacy of UFT against several types of malignant tumors, including bladder cancer [9-14]. In this study, therefore, a randomized clinical trial was conducted in order to assess the significance of the adjuvant administration of UFT for patients who underwent TUR and were subsequently diagnosed as having NMIUCB. In this trial, we also analyzed the impact of UFT therapy on changes in the serum level of its metabolite $\gamma$-butyrolactone (GBL), a potent inhibitor of angiogenesis [15], in addition to those of major angiogenic factors, including vascular endothelial growth factor (VEGF), basic fibroblast growth factor (bFGF), platelet-derived growth factor (PDGF) and interleukin-8 (IL-8) [16].

\section{Patients and Methods}

The population of this study consisted of patients who were at least 18 years old and underwent complete TUR of pathologically documented NMIUCB. Key eligibility criteria for this study included an Eastern Cooperative Oncology Group performance status of 0 or 1 , adequate hematologic, coagulation, hepatic, renal and cardiac functions, and the absence of perioperative anticancer therapy. Patients were ineligible if they had carcinoma in situ or T1G3 disease and a history of malignant disease during the preceding 12 months. Written informed consent to enter this study was obtained from all patients, and the study design was approved by the Research Ethics Committee of our institution.

A total of 48 patients who were judged to be eligible were included and randomly assigned to either the adjuvant therapy group (group A) or the control group (group B). In group A, 2 weeks after TUR, UFT was orally given at a dose of at least 300 $\mathrm{mg} / \mathrm{d}$ for 12 months, while no adjuvant therapy was performed in group B. Toxicities associated with the administration of UFT were graded according to the NCI Common Toxicity Criteria, and if an adverse event greater than grade 1 occurred, the dose of UFT was reduced. Postoperative follow-up examinations were carried out as follows: cystoscopy and urinary cytological examination were performed every 3 months for 3 years after TUR, and then every 6 months until 5 years after TUR, and intravenous pyelography was performed every 6 months until 3 years after TUR and then annually until 5 years after TUR. On the detection of tumors or hyperemic mucosa by cystoscopy and/or positive findings on urinary cytology, transurethral biopsy of the abnormal region and/ or TUR of the tumor were carried out.

To assess the effects of UFT therapy on its metabolite and major angiogenic factors, serum samples were obtained from all patients approximately 4 weeks after their inclusion in this trial, and the sample collection in group A was also done at 2 hours after the administration of UFT. Serum levels of GBL were determined by employing gas chromatography mass spectrometry as previously described [17]. For the measurement of serum concentrations of VEGF, bFGF, PDGF and IL-8, commercially available sandwich enzyme-linked immunoassay kits were employed according to the manufacturer's recommendations (R\&D Systems, Minneapolis, MN, USA).

Differences between groups A and B were compared using the chi-square test or unpaired-t test. Intravesical recurrence-free survival rates were calculated using the Kaplan-Meier method, and the difference was determined by the log-rank test. The prognostic significance of certain factors was assessed by employing the Cox proportional hazards regression model. All statistical calculations were performed using Statview 5.0 software (Abacus Concepts, Inc., Berkley, CA, USA), and probability (p) values less than 0.05 were considered significant.

\section{Results}

Of the 48 eligible patients, 27 and 21 were assigned to groups $\mathrm{A}$ and $\mathrm{B}$, respectively. There were no significant differences in several clinicopathological parameters between groups A and B (table 1).

During the observation period of this study (median 24 months, range 11 - 42 months), intravesical recurrence was detected in 14 patients in group $\mathrm{A}(51.9 \%)$ and 9 patients in group B $(42.9 \%)$. There was no significant difference in intravesical recurrence-free survival between these 2 groups (fig. 1).

Adverse events associated with adjuvant UFT therapy were observed in $6(22.2 \%)$ of the 27 patients assigned to group A, including gastrointestinal symptoms in 5 and thrombocytopenia in 1. However, interruption of UFT therapy was not required in any patient, while dose reduction from 600 to $300 \mathrm{mg} / \mathrm{d}$ was necessary in 2 patients with gastrointestinal symptoms corresponding to grade 2.

As shown in figure $2 \mathrm{~A}$, mean serum levels of GBL in groups A and B were 37.5 and $21.1 \mathrm{ng} / \mathrm{ml}$, respectively, and there was a significant difference in the serum GBL level between these 2 groups. However, there were no significant differences in serum concentrations of VEGF, bFGF, PDGF and IL- 8 between groups A and B (fig. 2B-E). 
Table 1. Patient characteristics in group $A(n=27)$ and $B(n=$ 21)

\begin{tabular}{lccc}
\hline & Group A & Group B & $\mathrm{p}$ \\
\hline Age, years & $64.8 \pm 9.7$ & $72.5 \pm 9.0$ & 0.14 \\
Gender & & & 0.96 \\
$\quad$ Male & 23 & 18 & \\
$\quad$ Female & 4 & 3 & \\
Multiplicity & & & 0.72 \\
$\quad$ Solitary & 14 & 12 & \\
$\quad$ Multiple & 13 & 9 & \\
Size, cm & & & 0.78 \\
$\quad$ Less than 3 & 19 & 14 & \\
$\quad$ or greater & 8 & 7 & \\
History of upper urinary tract caner & & & 0.47 \\
$\quad$ Positive & 9 & 5 & \\
$\quad$ Negative & 18 & 16 & \\
Grade & & & 0.65 \\
$\quad$ 1 & 8 & 5 & \\
$\quad$ Ttage & 19 & 19 & \\
$\quad$ Ta & & & 0.78 \\
$\quad$ T1 & 21 & 17 & \\
\hline
\end{tabular}

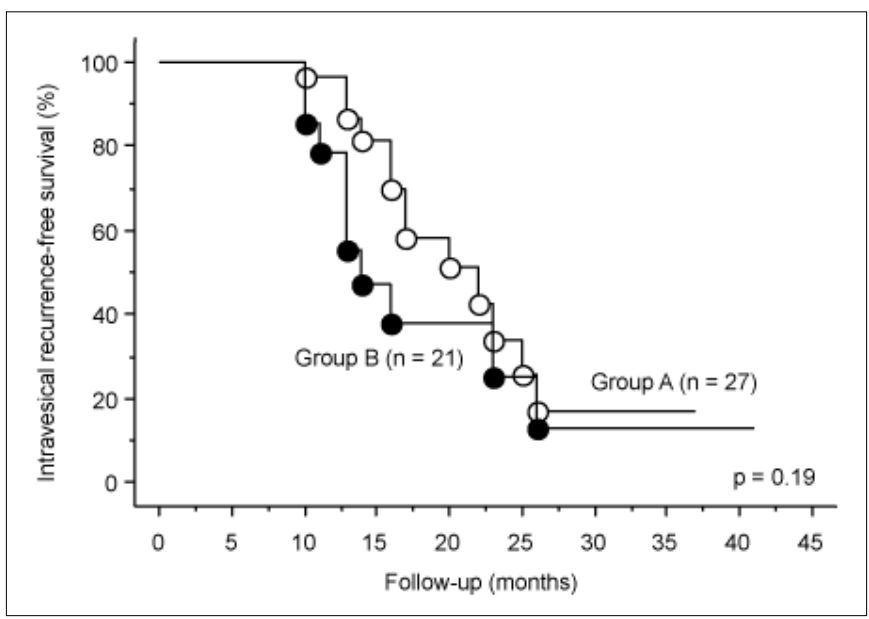

Fig. 1. Intravesical recurrence-free survival of patients with NMIUCB in groups A and B.

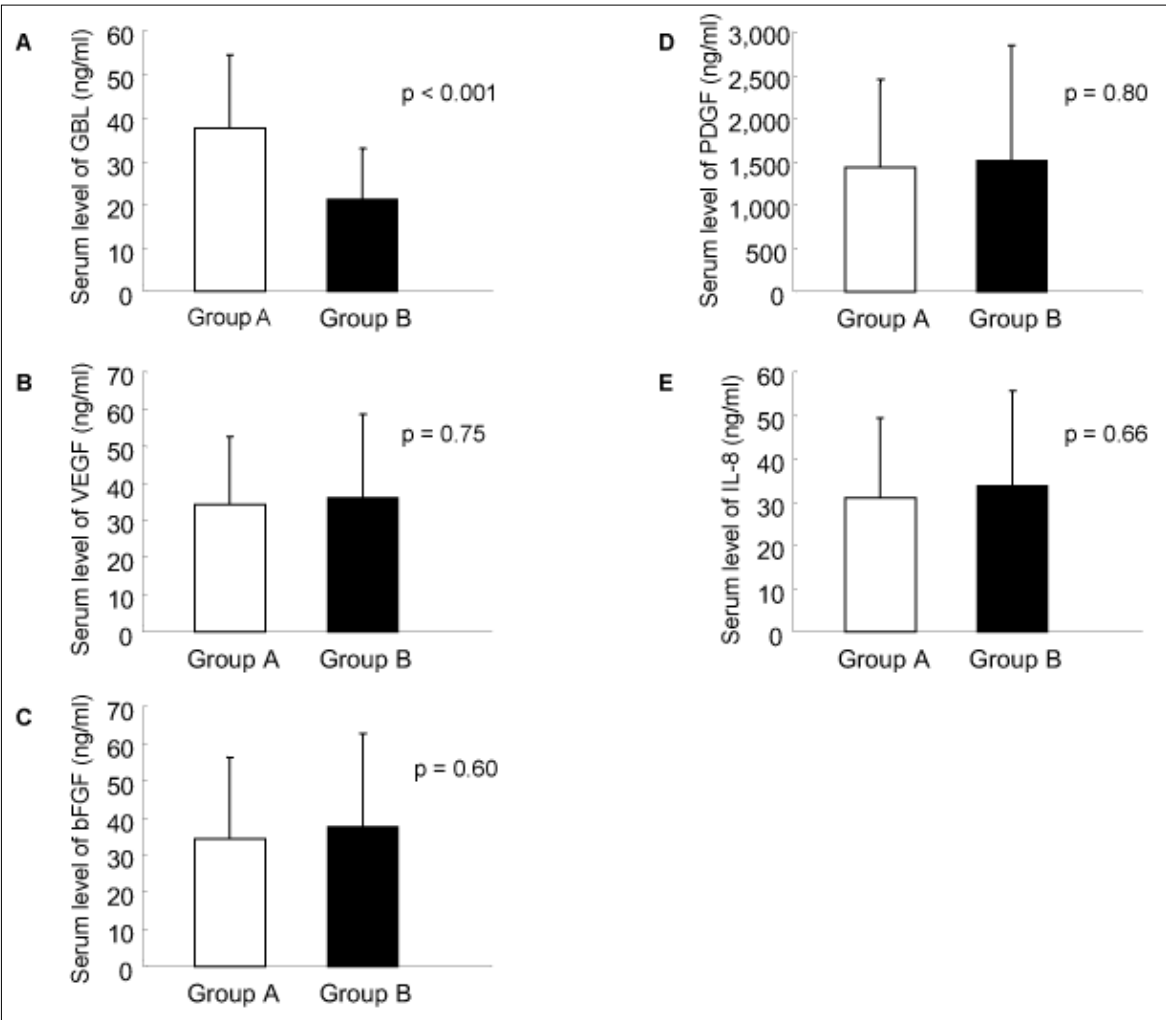

Fig. 2. Comparison of serum levels of A: GBL; B: VEGF; C: bFGF; D: PDGF; E: IL-8 between groups A and B. 
Table 2. Univariate and multivariate analysis of the associations between various parameters and intravesical recurrence-free survival

\begin{tabular}{|c|c|c|c|c|}
\hline & \multicolumn{2}{|c|}{ Univariate analysis } & \multicolumn{2}{|c|}{ Multivariate analysis } \\
\hline & Hazard ratio & $\mathrm{p}$ & Hazard ratio & $\mathrm{p}$ \\
\hline Gender, male vs. female & 0.97 & 0.88 & & \\
\hline Multiplicity, solitary vs. multiple & 2.07 & 0.031 & 1.22 & 0.2 \\
\hline Size, $<3$ vs. $\geq 3 \mathrm{~cm}$ & 2.23 & 0.015 & 1.98 & 0.033 \\
\hline T stage, Ta vs. T1 & 1.54 & 0.12 & & \\
\hline Adjuvant UFT therapy, yes vs. no & 0.83 & 0.46 & & \\
\hline Serum GBL level, high vs. low ${ }^{\mathrm{a}}$ & 0.79 & 0.35 & & \\
\hline Serum VEGF level, high vs. low ${ }^{\mathrm{a}}$ & 0.94 & 0.89 & & \\
\hline Serum BFGF level, high vs. low ${ }^{\mathrm{a}}$ & 1.11 & 0.78 & & \\
\hline
\end{tabular}

${ }^{\text {a } D e t e r m i n e d ~ b a s e d ~ o n ~ t h e ~ m e d i a n ~ v a l u e ~ o f ~ e a c h ~ m o l e c u l e ~ a s ~ a ~ c u t-o f f ~ p o i n t . ~}$

To analyze factors significantly related to postoperative intravesical recurrence, univariate and multivariate analyses were performed. Multiplicity and tumor size were identified as significant predictors on univariate analysis, however, the remaining factors, including adjuvant therapy and serum levels of GBL, VEGF, bFGF, PDGF and IL-8, had no significant impact on intravesical recurrence. In addition, tumor size appeared to be independently associated with intravesical recurrence on multivariate analysis (table 2).

\section{Discussion}

To date, a number of investigators have evaluated the usefulness of intravesical instillation therapy in order to delay or prevent intravesical recurrence of NMIUCB following TUR; however, there are several limitations regarding this therapy [4-7]. For example, intravesical instillation itself involves a comparatively invasive procedure and usually takes approximately 1 hour to keep an agent in the bladder. In addition, it has been well documented that more than $10 \%$ of patients who receive intravesical therapy experience complications, including uncommon but severe adverse events [6]. Also, from an oncological viewpoint, there has been no report of intravesical therapy leading to a significant improvement of overall survival in patients with NMIUCB $[4,5]$. Collectively, these findings suggest that it is necessary to develop an effective adjuvant therapy with an orally available agent following TUR of NMIUB.

UFT, the combination of uracil and tegafur in a $4: 1$ molecular ratio, is an oral anticancer agent showing selective activity, because biochemical modulation by uracil enhances fluorouracil concentration more specifically in tumor tissues than that in normal tissues [8]. A number of studies have reported the usefulness of UFT for a wide variety of malignant diseases, such as lung, breast, gastric and prostate cancer [9-11]. For bladder cancer, the efficacy of UFT has been widely investigated as a therapeutic as well as prophylactic agent, and some of these studies have demonstrated that treatment with UFT is tolerable and effective in delaying progression and preventing recurrence in patients with bladder cancer [12-14]. Considering these findings, the significance of adjuvant UFT therapy in the prevention of intravesical recurrence in patients undergoing TUR for NMIUCB was evaluated.

In this series, there were no significant differences in several clinicopathological parameters between the treatment and control groups. In addition, there was no significant difference in the intravesical recurrence-free survival between these 2 groups. To our knowledge, there has been only one study evaluating the efficacy of UFT 
as adjuvant therapy for NMIUCB. However, in this previous study, Kubota et al. [13] reported a significantly more favorable recurrence rate in patients with NMIUCB who received adjuvant UFT therapy compared to that in those without any anticancer therapy. This discrepancy may be explained by the different characteristics of patients included in these 2 studies. In fact, Kubota et al. [13] showed a marked prophylactic effect of adjuvant UFT therapy in patients with a single small tumor less than $1 \mathrm{~cm}$ in diameter, while approximately 50 and $35 \%$ of patients included in the present study had multiple tumors and large tumors greater than $3 \mathrm{~cm}$ in diameter, respectively.

UFT has been shown to be resolved into GBL and its acid form, $\gamma$-hydroxybutyric acid, which exists in chemical equilibrium under physiological conditions [18]. Recently, GBL was demonstrated to inhibit tumor-induced angiogenesis based on a dorsal air sac assay performed in mice with 5 kinds of cancer cell lines [15]. In the present study, the serum level of GBL in the treatment group was significantly greater than that in the control group. However, there were no significant differences in the serum level of VEGF in addition to those of other major angiogenic factors, including bFGF, PDGF and IL-8, between these 2 groups. Based on our present findings, therefore, the anti-angiogenic activity of GBL might be exerted independently of major angiogenic factors, including VEGF. Nagai et al. [17] however, reported that serum GBL levels, which increased following the administration of UFT, inhibited the angiogenesis induced by VEGF in patients with advanced cervical cancer. That is, VEGF protein expression in approximately $60 \%$ of cervical cancer tissues was decreased by UFT therapy, and there was an inverse correlation between changes in serum GBL and VEGF levels after treatment with UFT. This is likely to be affected by several factors, such as the dose of UFT, origin of the cancer and method of assay, and whether VEGF is involved in the inhibition of angiogenesis induced by GBL. Accordingly, further studies are necessary to clarify this issue.

Here, we would like to emphasize the limitations of this study. First, a sample size of 48 patients for such a common disease as NMIUCB is not large enough, and the observation period in this series might have been too short to evaluate the actual recurrence rate. Second, the effects of UFT on angiogenesis were evaluated based on serum levels of angiogenic factors, which may not precisely reflect the production of these factors by tumor cells. Finally, this study failed to show the preventive effect of the adjuvant use of UFT on intravesical recurrence

UFT for Non-Muscle Invasive Bladder Cancer following TUR of NMIUCB despite the significant increase in GBL induced by UFT T. Therefore, it is necessary to develop a more optimal schedule of adjuvant UFT therapy to effectively reduce the proportion of patients developing intravesical recurrence. Considering several studies showing the important role of angiogenesis in the intravesical recurrence of NMIUCB $[19,20]$, one possible approach is to increase the dose of UFT up to 600 $\mathrm{mg} / \mathrm{d}$ for all patients in order to achieve a more potent inhibitory effect on the angiogenic activity of NMIUCB. In fact, Shida et al. [14] reported that the proportion of responders in patients with bladder cancer receiving 600 $\mathrm{mg} / \mathrm{d}$ of UFT was twice as high as that in those receiving $300 \mathrm{mg} / \mathrm{d}$ of UFT in Phase II studies.

In conclusion, adjuvant UFT therapy in patients with NMIUCB significantly induced its metabolite, GBL, accompanied by no significant changes in major angiogenic factors, including bFGF, PDGF and IL-8, and failed to reduce postoperative intravesical recurrence. Therefore, we should consider enhancing the anti-angiogenic effect of GBL by using an alternative administration schedule of UFT to more effectively prevent intravescial recurrence of NMIUCB following TUR. 


\section{References}

1 Oosterlinck W: The management of superficial bladder cancer. BJU Int 2001;87:135140.

- 2 Hisataki T, Miyao N, Masumori N, Takahashi A, Yanase M, Itoh N, Tsukamoto T: Risk factors for multiple intravesical recurrences of superficial bladder cancer. Urology 2001;58:935-939.

-3 Sakai I, Miyake H, Harada K, Hara I, Inoue TA, Fujisawa M: Analysis of factors predicting intravesical recurrence of superficial transitional cell carcinoma of the bladder without concomitant carcinoma in situ. Int $\mathbf{J}$ Urol 2006;13:1389-1392.

4 Dobruch J, Herr H: Should all patients receive single chemotherapeutic agent instillation after bladder tumour resection? BJU Int 2009;104:170-174.

5 Shelley MD, Mason MD, Kynaston H: Intravesical therapy for superficial bladder cancer: a systematic review of randomized trials and meta-analyses. Cancer Treat Rev 2010;36: 195-205.

6 Jones JS: Routine use of perioperative intravesical chemotherapy after TURBT: against. Urology 2010;76:797-798.

7 Whelan P: Bladder cancer - contemporary dilemmas in its management. Eur Urol 2008; 53:24-26.

-8 Fujii S, Kitano S, Ikenaka K, Shirasaka T: Effect of co-administration of uracil or cytosine on the anti-tumor activity of clinical doses of 1-(2-tetrahydrofuryl)-5-fluorouracil and levels of 5-fluorouracil in rodents. Gann 1979; 70:209-214.
$\$ 9$ Taguchi T: Clinical application of biochemical modulation in cancer chemotherapy: biochemical modulation for 5-FU. Oncology 1997;54(suppl 1):12-18.

10 Kato H, Ichinose Y, Ohta M, Hata E, Tsubota $\mathrm{N}$, Tada H, Watanabe Y, Wada H, Tsuboi M, Hamajima N, Ohta M: A randomized trial of adjuvant chemotherapy with uracil-tegafur for adenocarcinoma of the lung. $\mathrm{N}$ Engl $\mathrm{J}$ Med 2004;350:1713-1721.

11 Miyake H, Hara I, Yamazaki H, Eto H: Clinical outcome of oral uracil/tegafur (UFT) therapy for patients with hormone refractory prostate cancer. Oncol Rep 2005;14:673676.

12 Kubota Y, Noguchi S, Hosaka M: UFT in bladder cancer. Oncology 1999;13(7 suppl 3):112-115.

13 Kubota Y, Hosaka M, Fukushima S, Kondo I: Prophylactic oral UFT therapy for superficial bladder cancer. Cancer 1993;71:1842-1845.

14 Shida K, Yamanaka H, Ito Y, Shimazaki J, Isaka S, Yokokawa M, Fukui I, Kawai H, Akimoto M, Yoshida K: Phase II study of UFT for malignant tumors of urinary organs. Jpn $\mathbf{J}$ Cancer Chemother 1984;11:1307-1314.

15 Yonekura K, Basaki Y, Chikahisa L, Okabe S, Hashimoto A, Miyadera K, Wierzba K, Yamada Y: UFT and its metabolites inhibit the angiogenesis induced by murine renal cell carcinoma, as determined by a dorsal air sac assay in mice. Clin Cancer Res 1999;5:21852191.
16 Eissa S, Swellam M, Labib RA, El-Zayat T, El Ahmady O: A panel of angiogenic factors for early bladder cancer detection: enzyme immunoassay and Western blot. J Urol 2009; 181:1353-1360.

17 Nagai N, Mukai K, Hirata E, Jin HH, Komatsu M, Yunokawa M: UFT and its metabolite gamma-butyrolactone (GBL) inhibit angiogenesis induced by vascular endothelial growth factor in advanced cervical carcinoma. Med Oncol 2008;25:214-221.

$18 \mathrm{Wu}$ AT, Au JL, Sadee W: Hydroxylated metabolics of R,S-1-(tetrahydro-2-furanyl)5 -fluorouracil (Ftorafur) in rats and rabbits. Cancer Res 1978;38:210-214.

19 Agrawal U, Mishra AK, Salgia P, Verma S, Mohanty NK, Saxena S: Role of tumor suppressor and angiogenesis markers in prediction of recurrence of non muscle invasive bladder cancer. Pathol Oncol Res 2011;17: 91-101.

20 Miyake H, Hara I, Yamanaka K, Gohji K, Arakawa S, Kamidono S: Elevation of serum level of vascular endothelial growth factor as a new predictor of recurrence and disease progression in patients with superficial urothelial cancer. Urology 1999;53:302-307. 